\title{
Time-frequency voiced and unvoiced excitation models for harmonic speech systems
}

\author{
Miguel Arjona Ramírez
}

\begin{abstract}
A time-frequency voiced model (TFVEX) is proposed for the excitation of a harmonic autoregressive (AR) wideband speech analysis-synthesis system, which is integrated with a previously proposed unvoiced time-frequency model in the time-frequency unvoiced-voiced excitation (TFUVEX) framework. The voiced component model has low time resolution defined by the concentration of the excitation signal distribution in the modulation domain while the time-frequency unvoiced excitation (TFUNEX) model has cycle time discrimination with lower amplitude resolution and while the frequency resolution for both models is an octave in the high time resolution domain. The speech reconstructed by the compound TFUVEX model is rated above the speech degraded by a modulated noise reference unit (MNRU) at $25 \mathrm{~dB}$ in listening tests while yielding a parametric compression of over ten times.
\end{abstract}

Index Terms- speech analysis, speech coding, sparse representations, modulation transform, time-frequency analysis, voicedunvoiced decision

\section{INTRODUCTION}

$\mathbf{H}$ ARMONIC speech representations have been used for coding at medium to low bit rates [1] even when they fail to achieve perfect reconstruction. However, it is desirable to have a nearly perfect reconstruction (NPR) frontend representation since it is not bounded in performance at high rates.

The classification of speech segments into voiced and unvoiced classes is important for speech modification and speech coding since they are processed differently. Besides, sparse speech representations are useful for source separation [2] and, if conceptually designed, they may be the basis for pattern playback in signal processing education [3].

Usually, a model is fit to the voiced harmonic amplitudes [1], [4] for ease of manipulation and often as an intermediate stage in coding. Somehow unexpectedly, an unvoiced model is important in making a harmonic system deliver natural-sounding speech [1], [5] or, for that matter, any synthesized audio signal [6], in particular music [7], [8] since any instrumental performance requires some random fluctuations in order to sound natural.

In this work a voiced model is proposed for an NPR frontend representation [9] and this model fits into a framework that includes the unvoiced model previously proposed [10] so that sparse speech representations may be achieved. Unlike usual harmonic representations which apply hard decision for voiced and unvoiced speech classification in the time and/or in the frequency domain, the voiced-unvoiced decision

This work is supported by Conselho Nacional de Desenvolvimento Científico e Tecnológico (CNPq) under Grant no. 307633/2011-0 and by Fundação de Amparo à Pesquisa do Estado de São Paulo (FAPESP) under Grant no. 2012/24789-0. in this analysis-synthesis system (ASyS) is equivalent to a soft decision because the separation is implemented in the modulation domain.

The nature of the voiced-unvoiced classification will be appreciated after the description of ASyS, when the criteria for classifying and segmenting speech excitation into voiced part and unvoiced part are presented in Section II. The voiced model (TFVEX) is developed in Section III upon specific time-frequency features of voiced excitation, which are to be contrasted with those of the unvoiced model (TFUNEX) [10] that follows in Section IV. Then, the compound voicedunvoiced model (TFUVEX) is presented in Section V with the introduction of the spectral weighting in the pitch-synchronous domain. Finally, the three excitation models are assessed in Section VI and remarks are drawn in conclusion.

\section{SPEECH EXCITATION CLASSIFICATION AND SEPARATION}

Sinusoidal representations of speech are related to harmonic speech systems [11]. In fact, sinusoidal models may even represent an inharmonic signal segment $s(n)$, which may be expressed by the modeling equation

$$
\tilde{s}(n)=\sum_{l=1}^{K} A_{l} \cos \left(\omega_{l} n+\phi_{l}\right),
$$

whose parameters are the frequencies $\omega_{l}$, the amplitudes $A_{l}$ and the phases $\phi_{l}$ for $l=1,2, \ldots, K$. When the signal segment $s(n)$ is periodic, the model may be simplified to the harmonic expression

$$
\tilde{s}(n)=\sum_{l=1}^{K} A_{l} \cos \left(l \omega_{0} n+\phi_{l}\right),
$$

which has a simplified frequency description by having all frequencies satisfying $\omega_{l}=l \omega_{0}$, for $l=1,2, \ldots, K$, that is, they are harmonically related to the fundamental frequency $\omega_{0}=2 \pi / p_{0}$, where $p_{0}$ is the pitch period.

More advanced implementations of sinusoidal coders derive their amplitudes and phases from the frequency response $H\left(e^{j \omega}\right)$ of a synthesis filter $H(z)$ so that the synthesized signal segment is represented as

$$
\tilde{s}(n)=\sum_{l=1}^{K} \operatorname{Re}\left\{H\left(e^{j l \omega_{0}}\right) \exp \left(j l \omega_{0} n\right)\right\}
$$

This terminal model may be further decomposed into a model for the excitation signal segment

$$
\tilde{d}(n)=\sum_{l=1}^{K} \operatorname{Re}\left\{\exp \left(j l \omega_{0} n\right)\right\}
$$


which is applied to the input of the synthesis filter.

If we are now to model the excitation signal directly, we must obtain it by filtering the speech signal segment $s(n)$ by the analysis filter, which is the inverse filter

$$
A(z)=\frac{1}{H(z)}
$$

For ASyS, the analysis filter is the linear prediction (LP) whitener block in Fig. 1. It is a spectrum whitener because the frequency components at its output tend to be very near in amplitude. In fact, for the source-filter sinusoidal excitation model in Eq. (4), the harmonic amplitudes are exactly the same.

The speech excitation signal for ASyS is obtained from the speech signal $s(n)$ as the linear prediction (LP) residual signal $r(n)$ in Fig. 1. This whitened signal has a lower dynamic range in the time-frequency domain [12]. The order of this AR model is consistent with sound propagation in the air inside a typical $17-\mathrm{cm}$ long male vocal tract, which in a neutral configuration has one formant per kilohertz in its spectrum [13]. Additionally, two poles may be included to account for glottal and radiation effects so that, overall, typical AR models orders are 10 and 12 for narrowband speech [14] and 16 for wideband speech [15].

But Rosenberg argues that, for source spectra of natural speech, the typical asymptotic spectral decay is $12 \mathrm{~dB} /$ oct [16], which requires two units of order to be assigned in accounting for the glottal pulse shape so that our count for wideband speech speech would rise to 17 th order. For ASyS, at a sampling rate of $16 \mathrm{kHz}, 18$ th-order AR modeling has been found adequate.

The signal is windowed with square-root von Hann windows, which support perfect reconstruction under transform analysis and synthesis. The window length is $20 \mathrm{~ms}$, which is compatible with quasistationarity for LP analysis of speech [17]. Additionally, a frame rate of $400 \mathrm{~Hz}$ allows for high temporal resolution.

The prediction residual signal is then time-warped to $r_{w}(\nu)$, for $\nu \in \mathbb{Z}$, by means of bandlimited sinc functions in order to hold the pitch period length constant at $P_{0}$ samples [10] as depicted in Fig. 1 whereas the original pitch track $p(n)$ is separated. This allows intrapitch variations to be captured by the pitch-synchronous transform in Fig. 2 due to the constant pitch period after the time warper while preserving the pitch period information in the pitch track for further synthesis.

Consequently, at this point the signal power is more evenly distributed in both the time and the frequency domains as illustrated in Fig. 2, where the time-frequency distributions of the power in the speech signal and in the residual signal are represented by a spectrogram and by the intensity of a pitch-synchronous transform (PST), respectively.

The time-warped residual undergoes a pitch-synchronous transform, which is a modulated lapped transform (MLT) [18] that produces the harmonic tracks

$$
c_{l}(k)=\sum_{\nu=k P_{0}}^{(k+2) P_{0}-1} r_{w}(\nu) \phi_{l}\left(\mu_{k}(\nu)\right)
$$

for pitch cycles $k \in \mathbb{Z}$, harmonic indices $l \in\left\{0,1, \ldots P_{0}-1\right\}$ and local warped time $\mu_{k}(\nu)=\nu+[4-(k \bmod 4)] 2 P_{0}$, where the basis functions are

$$
\phi_{l}\left(\mu_{k}\right)=\sqrt{\frac{2}{P_{0}}} \cos \left[\frac{2 \pi\left(l+\frac{1}{2}\right)\left(\mu_{k}-\frac{P_{0}}{2}+\frac{1}{2}\right)}{2 P_{0}}\right] w_{k}\left(\mu_{k}\right) \text {. }
$$

The PST is evaluated at each pitch cycle over a two-cycle long window $w_{k}\left(\mu_{k}\right)$, which is a modified square-root von Hann window

$$
w_{k}\left(\mu_{k}(\nu)\right)=\sqrt{\frac{1}{2}-\frac{1}{2} \cos \left[\frac{2 \pi\left(\mu_{k}(\nu)+\frac{1}{2}\right)}{2 P_{0}}\right]}
$$

for the $k$ th pitch cycle. In fact, any smooth symmetric window could be used as long as its sum of squares is $P_{0}$ so that it satisfies the perfect reconstruction condition.

The modulation transforms (MTs) of the resulting PST time tracks, for $l \in\left\{0,1, \ldots P_{0}-1\right\}$, are obtained in modulation segment $m$ as

$$
e_{q l}(m)=\sum_{k=k_{0 m}}^{k_{0 m}+Q_{m}-1} c_{l}(k) \psi_{m q}\left(\chi_{m}(k)\right)
$$

where $Q_{m}$ is the segment length with initial cycle index $k_{0 m}$, local cycle index $\chi_{m}(k)=k-\sum_{i=0}^{m-1} Q_{i}$ and type-II DCT [19], [20] basis functions

$$
\psi_{m q}\left(\chi_{m}\right)=\sqrt{\frac{2}{Q_{m}}} \gamma_{q} \cos \left(\frac{q \pi}{Q_{m}} \chi_{m}+\frac{q \pi}{2 Q_{m}}\right) w_{m}\left(\chi_{m}\right)
$$

for modulation frequencies (MFs) $q \in\left\{0,1, \ldots, Q_{m}-1\right\}$, where coefficient $\gamma_{q}$ is defined as

$$
\gamma_{q}=\left\{\begin{array}{lll}
\frac{1}{\sqrt{2}} & \text { if } q=0 & \bmod Q_{m} \\
1 & \text { if } q \neq 0 & \bmod Q_{m}
\end{array}\right.
$$

and $w_{m}\left(\chi_{m}(k)\right)$ represents the rectangular window for the $m$ th modulation segment, which is supported within the interval $\left\{k_{0 m}, k_{0 m}+1, \ldots, k_{0 m}+Q_{m}-1\right\}$.

It should be noted that the analysis process shown in Fig. 1 can be inverted perfectly for obtaining the excitation signal back. The MT and the PST can be inverted perfectly within the numerical precision of the system and the LP-whitener plus time-warper cascade couple can be inverted with segmental SNR of around $50 \mathrm{~dB}$ for obtaining the reconstructed speech signal as verified in our experiments. In fact, reconstruction signal-to-noise ratios in the $60 \mathrm{~dB}$ to $70 \mathrm{~dB}$ range are reported to have been obtained with this subsystem [9].

The concentration measure of the time-frequency distribution (CMTFD) used for the modulation transform is the unnormalized modified Zakai's entropy

$$
C_{E}\left(m, Q_{m}\right)=-\sum_{q=0}^{Q_{m}-1} \sqrt{\sum_{l=0}^{P_{0}-1} e_{q l}^{2}(m)}
$$

where the square root replaces the original log function [21] while preserving the convexity of the measure. The dimension of the DCT space is increased one step further if its CMTFD satisfies the inequality

$$
C_{E}\left(m, Q_{m}+1\right) \geq C_{E}\left(m, Q_{m}\right)+C_{E}(m+1,1)-\lambda
$$




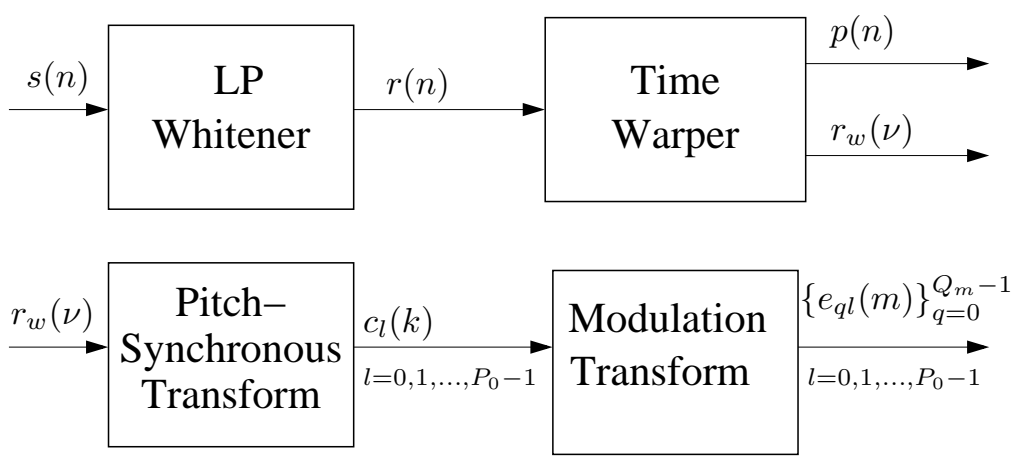

Fig. 1. Block diagram of the analysis process in ASyS.

where $C_{E}\left(m, Q_{m}\right)$ is the CMTFD for the current modulation segment and $C_{E}(m+1,1)$ is the CMTFD for the next singlecycle segment while $\lambda$ is the difference CMTFD threshold, which should be unity for wideband speech with unit-variance residual signal. By increasing the modulation segment length in unit steps, the actual length $Q_{m}$ is reached when inequality (12) fails to hold.

The baseline for voiced-unvoiced separation is a quite periodic residual whose energy lies completely in the DC modulation coefficient for all harmonic tracks. In general, the most energetic voiced coefficients are at low modulation bands [9] and speech itself has its energy concentrated in rather low modulation frequencies [22]. In fact, for a sequence of nonidentical pitch cycles, it has been found that the lower

$$
q_{v m}=\max \left\{3,\left\lfloor 0.2 Q_{m}\right\rfloor\right\}
$$

MT coefficients represent the voiced part while the rest describe the unvoiced part [10]. Therefore, this voiced-unvoiced separation criterion effectively sets $q_{v m}-1$ as the voicing cutoff frequency for modulation segment $m$.

Finally, transients are envelope-detected and modeled like the voiced part. As a result, the average modulation segment length is around twelve pitch cycles and may be controlled by the CMTFD threshold. Further, the number of MFs in the unvoiced region is noted to be rather larger than that in the voiced region due to the voiced-unvoiced separation criterion.

\section{THE TIME-FREQUENCY VOICED EXCITATION MODEL}

The TFVEX model is based on the concept that a voiced model can have lower time resolution [1] and must have greater spectral amplitude resolution. In addition, a highfidelity representation for the low-frequency PST tracks emphasizes the harmonic structure.

The time resolution for the TFVEX model is the varying modulation segment length since it is a good estimate of the range of local stationarity. An algebraic sign applied to each local standard deviation estimates provides adequate spectral amplitude resolution.

Moreover, the representation will be more useful if it can be controlled by an easily manageable set of parameters. The third-octave band resolution used in audio analysis has motivated the clustering of frequency bands into nine bands. For wideband speech, the $L_{h}=P_{0}=256$ modulation

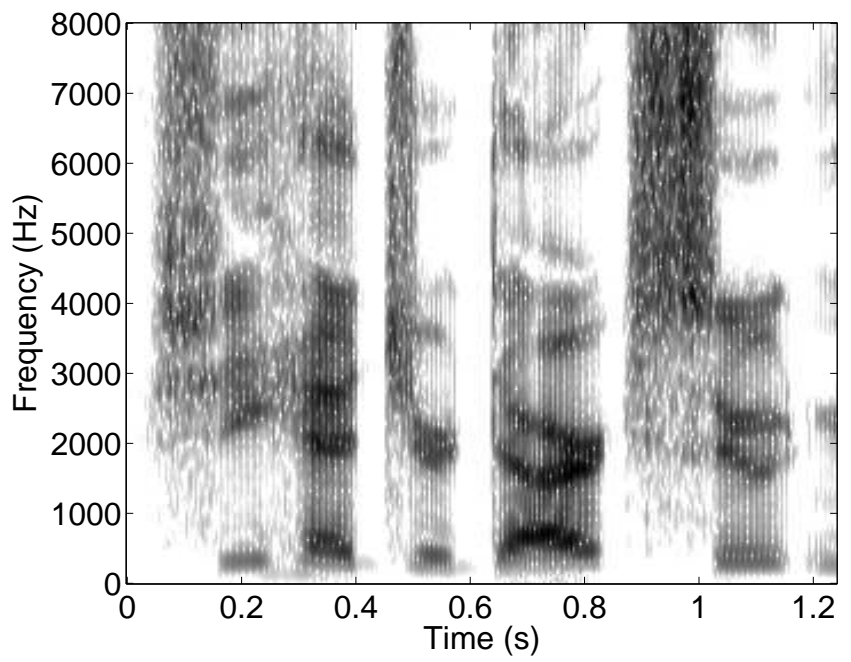

(a) Wideband spectrogram.

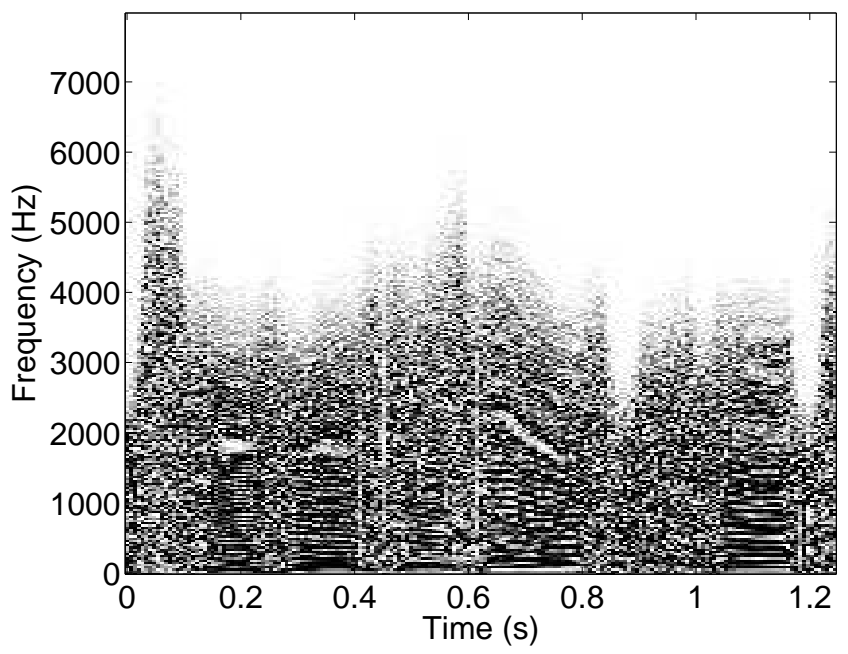

(b) Pitch-synchronous transform intensity plot.

Fig. 2. Wideband spectrogram of the speech signal and PST intensity plot of the prediction residual for the phrase "She had your dark suit...", uttered by a male speaker.

tracks of same MF are assigned to 9 octave bands that hold $1,1,2,4, \ldots, 128$ tracks, respectively.

But precision is not compromised since low-frequency fidelity is achieved by including in full precision the voiced 
MT coefficients for the lower compound band $B_{d}=\{l\}_{l=0}^{3}$. In this way, the model can reach on average sixth-octave resolution at low frequency. This happens to be so because the constant pitch period $P_{0}=256$, or fundamental frequency $F_{0}=31.25 \mathrm{~Hz}$, after time warping achieves a 6.4-fold increase in resolution for an average fundamental frequency of $200 \mathrm{~Hz}$.

The total number of bands has been chosen to approximate one band per octave at the lower fundamental frequency of 31.25 Hz. This clustering of PST tracks has a decreasing resolution as the frequency increases, which is consistent with hearing perception and has been found adequate in experiments.

For the set of upper five PST bands $B_{u}=\bigcup_{b=4}^{8} B_{b}$, the estimated MT voiced coefficient variance is evaluated as the mean square modulation intensity over its octave band by

$$
\left(\hat{\sigma}_{q b}^{(v)}(m)\right)^{2}=\frac{1}{2^{b-1}} \sum_{l=2^{b-1}}^{2^{b}-1}\left(e_{q l}^{(v)}(m)\right)^{2}
$$

for each upper band $B_{b}=\left\{2^{b-1}, 2^{b-1}+1, \ldots, 2^{b}-1\right\}$ for $b=4,5, \ldots, 8$ and MFs in the voiced range $0 \leq q \leq q_{v m}-1$, where $q_{v m}-1$ is the highest $\mathrm{MF}$ in the voiced region for modulation segment $m$.

Given the structure above, MT coefficients for the voiced model are retained for the lower band and made equal to the estimated variances affected by the original signs for the upper bands, that is,

$$
\hat{e}_{q l}^{(v)}(m)= \begin{cases}e_{q l}^{(v)}(m) & l \in B_{d} \\ \operatorname{sign}\left(e_{q l}^{(v)}(m)\right) \hat{\sigma}_{q b}^{(v)}(m) & l \in B_{b} \subset B_{u}\end{cases}
$$

for $l=0,1, \ldots, P_{0}-1$ and $0 \leq q \leq q_{v m}-1$. This modeled modulation segment is built immune to spillover across the voiced-unvoiced boundary by additionally defining

$$
\hat{e}_{q l}^{(v)}(m)=0
$$

for MFs in the unvoiced region $q_{v m} \leq q \leq Q_{m}-1$ and harmonic tracks $l=0,1, \ldots, P_{0}-1$.

Therefore, the TFVEX model consists of 8 MT coefficients, 5 variances and 248 signs. Packing signs into sets of sixteen 16-bit coded values, it amounts to a total of 29 parameters per voiced MF.

The framework for the voiced model is consistent with the unvoiced model, proposed in [10] and outlined in Section IV. However, TFVEX and TFUNEX differ in their time-frequency footprints and their amplitude resolution within.

\section{THE TIME-FREQUENCY UNVOICED EXCITATION MODEL}

The TFUNEX model is based on the facts that an unvoiced model can have lower spectral resolution [1] and must have greater time resolution. The latter is fulfilled by allowing TFUNEX to have pitch cycle resolution. This is consistent with the observation that the noise in the voiced regions of speech is coherent with the voiced part [8], [5].

The estimated variances for the upper bands are

$$
\left(\hat{\sigma}_{b}^{(u)}(k)\right)^{2}=\frac{1}{2^{b-1}} \sum_{l=2^{b-1}}^{2^{b}-1}\left(c_{l}^{(u)}(k)\right)^{2}
$$

for $b=4,5, \ldots, 8$. They shape the pitch-synchronous model tracks

$$
c_{l}^{(0)}(k)= \begin{cases}c_{l}^{(u)}(k) & \text { for } l \in B_{d} \\ \hat{\sigma}_{b}^{(u)}(k) x_{l}(k) & \text { for } l \in B_{b} \subset B_{u}\end{cases}
$$

for $l=0,1, \ldots, P_{0}-1$ and $k$ in modulation segment $m$, where $x_{l}(k)$ are generated by independent and identically distributed zero-mean, unit-variance Gaussian processes.

These tracks are modulation-transformed to $e_{q l}^{(0)}(m)$, that lead to the final model, which is obtained as

$$
\hat{e}_{q l}^{(u)}(m)= \begin{cases}0 & \text { for } 0 \leq q \leq q_{v m}-1 \\ e_{q l}^{(0)}(m) & \text { for } q_{v m} \leq q \leq Q_{m}-1\end{cases}
$$

for $l=0,1, \ldots, P_{0}-1$, where $q_{v m}-1$ is the voicing cutoff frequency defined in Section II. In the PST domain, the unvoiced excitation model for modulation segment $m$ is $\hat{c}_{l}^{(u)}(k)$ for $k$ in modulation segment $m$ and harmonic tracks $l=0,1, \ldots, P_{0}-1$.

In total, the TFUNEX model consists of 13 parameters per pitch cycle, which are 8 PST coefficients and 5 variances. Since the number of unvoiced MFs is usually much greater than the number of voiced MFs as noted in closing Section II, the compression effected by TFUNEX is greater than that achieved by TFVEX and the finer time resolution necessary for the unvoiced model is not a heavy penalty. Besides, this explains in part why the unvoiced model has been proposed first [10].

\section{THE COMPOUND TIME-FREQUENCY UNVOICED-VOICED EXCITATION MODEL}

The TFVEX model proposed in Section III and the TFUNEX model described in Section IV can be linearly combined in the modulation domain, giving rise to TFUVEX, the compound unvoiced-voiced model

$$
\hat{e}_{q l}^{(u v)}(m)=\hat{e}_{q l}^{(v)}(m)+\hat{e}_{q l}^{(u)}(m)
$$

for modulation frequencies $0 \leq q \leq Q_{m}-1$ in the $m$ th modulation segment and harmonic tracks $0 \leq l \leq P_{0}-1$, where $\hat{e}_{q l}^{(v)}(m)$ is the voiced model modulation coefficient for MF $q$ of the $l$ th harmonic track given by Eq. (15) or (16) and $\hat{e}_{q l}^{(u)}(m)$ is the unvoiced model modulation coefficient for MF $q$ of the $l$ th harmonic track given by Eq. (19). This combination is actually a time-frequency juxtaposition due to the antialiasing operations carried out in the modulation domain in the determination of both models.

It is interesting to note that while the TFUVEX model juxtaposes the models for the voiced and for the unvoiced parts in the modulation domain for flexible processing, the unvoiced model in [8] consists of wavelet transforms around each harmonic peak, which provides for a better recombination.

The TFUNEX model may cause distortion upon reconstruction in the lower frequency region as found in Fig. 3(b) in comparison to Fig. 3(a). The spectral fitting performance for TFUVEX is illustrated in Fig. 3(c), where it can be seen to follow rather closely the lower magnitude envelope. This improved rendition with two models lends support to their virtuous integration. 


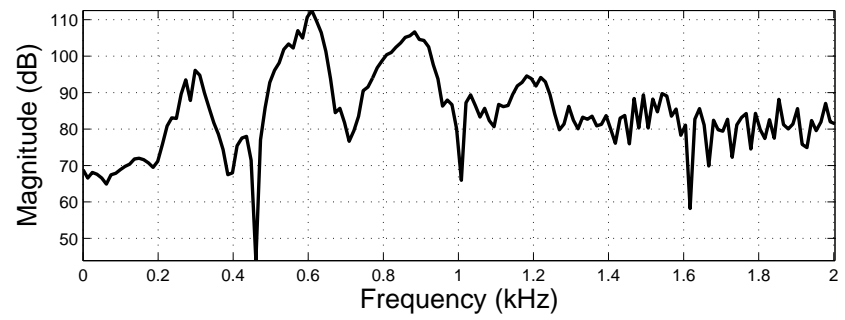

(a) Reference low-frequency speech signal spectrum.

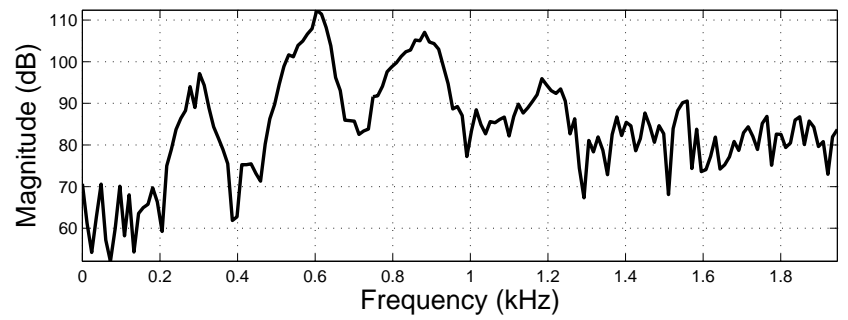

(b) Spectrum reconstructed with the TFUNEX model.

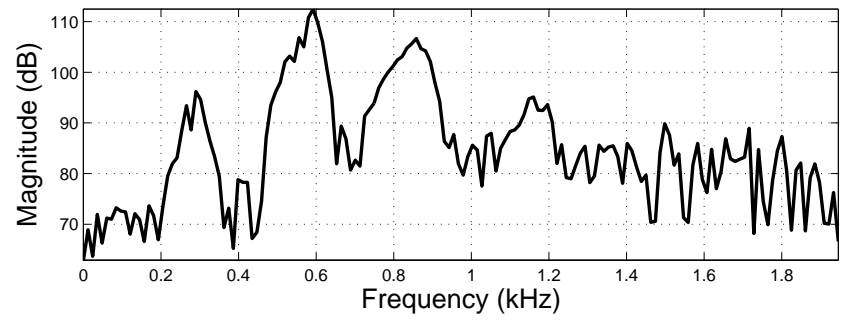

(c) Spectrum reconstructed with the TFUVEX model.

Fig. 3. Low-frequency speech signal spectrum (a) and its reconstruction with ASyS using the time-frequency unvoiced model and original voiced part (b) and using the compound time-frequency unvoiced-voiced model (c).

Further, a spectral enhancement has been found beneficial for better low frequency representation and smooth voicedunvoiced recombination. It is applied to the unvoiced model in the PST domain, where it does not interfere with the periodicity in the signal.

At first, a highpass filter with cyclic cutoff frequency $f_{c}=$ $11 / P_{0}$ is designed by means of the sinc function

$$
\operatorname{sinc}(n)=\frac{\sin (\pi n)}{\pi n}
$$

to have the impulse response

$$
w_{h}(n)=\left(1-2 f_{c}\right)(-1)^{n} \operatorname{sinc}\left[\left(1-2 f_{c}\right)(n-32)\right]
$$

for $n=0,1, \ldots, 64$.

The pitch-synchronous spectral weighting (PSSW) vector plotted in Fig. 4 is defined by sampling the frequency response of the highpass filter as

$$
w(l)=W_{h}\left(e^{j 2 \pi f_{l}}\right)
$$

at the cyclic frequencies $f_{l}=l /\left(2 P_{0}\right)$, for $l=0,1, \ldots, P_{0}-1$.

Finally, the enhanced model coefficients for unvoiced excitation are obtained by applying PSSW as

$$
\hat{c}_{l}^{(u) \prime}(k)=w(l) \hat{c}_{l}^{(u)}(k)
$$

for $l=0,1, \ldots, P_{0}-1$.

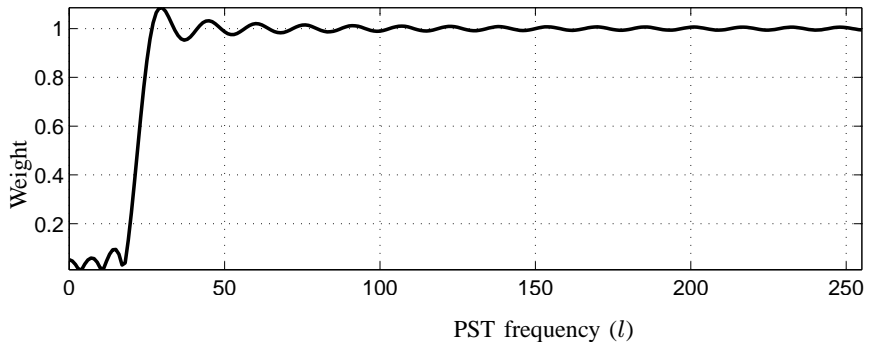

Fig. 4. Pitch-synchronous spectral weights.

\section{EXPERIMENTAL RESULTS}

For the listening tests, four male and four female utterances were chosen so that each speaker couple belongs to a different dialect region in the TIMIT database [23] for a total length of 448 thousand samples at a sampling rate of $16 \mathrm{kHz}$. Each signal was synthesized by ASyS in seven test conditions: the analyzed Voiced Part; the modeled Voiced Part plus the analyzed Unvoiced Part - TFVEX; the modeled Voiced Part singled out - TFVEX Alone; the analyzed Voiced Part plus the modeled Unvoiced Part - TFUNEX; the analyzed Voiced Part plus the modeled Unvoiced Part with PSSW emphasis TFUNEX-PSSW; the modeled Voiced Part combined with the modeled Unvoiced Part - TFUVEX; and the modeled Voiced Part plus the modeled Unvoiced Part with PSSW - TFUVEXPSSW.

Additionally, five control conditions were prepared for each signal, including the hidden reference and the following four anchors: modulated noise reference unit (MNRU) processed versions at $20 \mathrm{~dB}, 25 \mathrm{~dB}$, and $30 \mathrm{~dB}$ SNR levels; and a $3.5 \mathrm{kHz}$ lowpass-filtered version.

The twelve conditions above were presented to six female and six male listeners as a multi-stimulus with hidden reference and anchors (MUSHRA) test [24] whose results are displayed in Fig. 5.

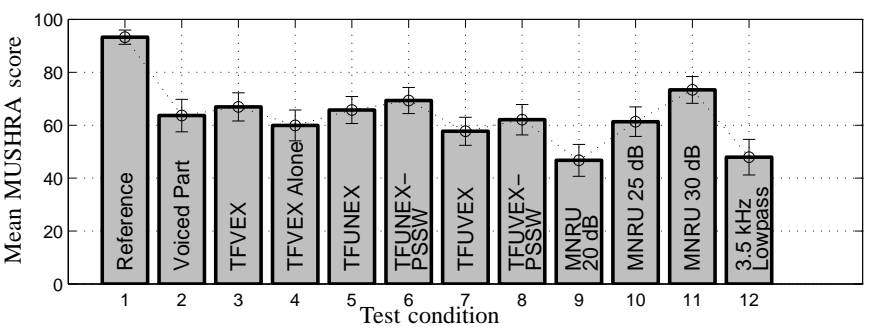

Fig. 5. Mean MUSHRA scores with $95 \%$ confidence intervals superimposed in subjective assessment of ASyS provided with TFVEX, TFUNEX and TFUVEX models.

It is striking that the TFVEX Model is rated somewhat above the analyzed Voiced Part and just a bit below when singled out, holding evidence to the high quality of the voiced model. Moreover, all single-model conditions are scored above $25 \mathrm{~dB}$ MNRU and the only two-model condition which rises above this level is TFUNEX-PSSW, underlining the distinctive contribution of the unvoiced model to higher fidelity.

Furthermore, the analyzed unvoiced part matters because "TFVEX" is better than "TFVEX Alone". But it is paradoxical 
that "TFUVEX" is rated slightly below "TFVEX Alone" as if the addition of the modeled unvoiced part would not improve the modeled voiced part. In fact, the modeled unvoiced part leaks into the voiced region while the analyzed unvoiced part does not. As shown in Section V, this interference is attenuated by means of the PSSW emphasis, but, in Fig. 5, its effect is not so outstanding since "TFUVEX-PSSW" is rated only slightly above "TFVEX Alone".

Besides, all test conditions provide wideband enhancement since their $Q$ values stand significantly above the $20 \mathrm{~dB}$ equivalent narrowband version.

The equivalent Q-value is the SNR in $\mathrm{dB}$ of the MNRUimpaired signal with the same score as a given condition and provides an inequivocal scale, with a universal meaning [25]. Scores are known to be cultural and they may acquire a universal meaning by their equivalent Q-values. Therefore, by using MNRUs at different levels, it is possible to make fair comparisons with other representation methods or test conditions. For instance, the adaptive multirate wideband (AMR-WB) coder has been rated at an equivalent Q-value of $24 \mathrm{~dB}$ when operating at $12.65 \mathrm{kbit} / \mathrm{s}$ [15].

In order to compare the performance under the same conditions, the AMR-WB coder has been applied to the same speech signals at the rates of $12.65 \mathrm{kbit} / \mathrm{s}$ and $23.85 \mathrm{kbit} / \mathrm{s}$ by means of the perceptual evaluation of speech quality (PESQ) [26], [27] with results shown in Fig. 6, where all time-frequency models perform above the coded speech.

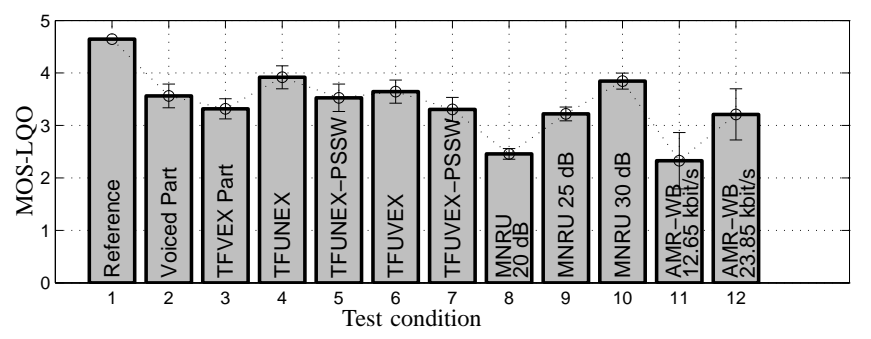

Fig. 6. Mean opinion scores (MOS-LQO - listening quality objective) with $95 \%$ confidence intervals superimposed in objective assessment of ASyS provided with TFVEX, TFUNEX, TFUVEX models compared to AMR-WB coded speech at two rates.

The test database has 4569 pitch cycles with 1644 voiced MFs for 1170 thousand raw coefficients. Since TFUNEX represents the unvoiced part of each cycle with 13 parameters, it uses 59 thousand parameters altogether. As for TFVEX, since it requires 29 parameters to represent each voiced MF across all harmonic tracks, it uses 48 thousand parameters altogether. Therefore, as detailed in Table I, the parametric compression ratios are about 9 for TFVEX over the voiced coefficient set and 1.5 overall, 13 for TFUNEX over the unvoiced coefficient set and 2.4 overall and 11 for TFUVEX over all coefficients.

\section{CONCLUSION}

A voiced excitation model is proposed for a harmonic time-frequency autoregressive speech analysis-synthesis system, which is integrated with a previously proposed unvoiced excitation model, giving rise to a voiced-unvoiced excitation model. The system features a time-frequency nearly perfect reconstruction (NPR) front-end for representation of the prediction residual that is transferred to a modulation domain within modulation segments of varying length based on a concentration measure of the time-frequency distribution. These modulation segments are the seat for voiced-unvoiced separation so that the voiced part and the unvoiced part are appropriately recombined all across the extent of the pitch-synchronous time-frequency transform. The length of the modulation segment also defines the time resolution for the TFVEX model while the TFUNEX model has pitch-cycle time resolution and lower amplitude resolution than TFVEX. Both model s have octave-band frequency resolution in the high time resolution domain so that they are conveniently combined into a compound TFUVEX unvoiced-voiced model with spectral weighting. The TFUVEX model generates speech of quality rated above that of speech degraded by $25 \mathrm{~dB}$ MNRU while providing a compression of over ten times relative to the NPR time-frequency representation that results in a lower rate parameter coding.

\section{REFERENCES}

[1] T. F. Quatieri, Discrete-Time Speech Signal Processing: Principles and Practice. Upper Saddle River: Prentice-Hall PTR, 2002, ch. 9.

[2] T. Arai, "Digital Pattern Playback for education in digital signal processing and speech science," in Proc. of IEEE Int. Conf. Acoust., Speech, Signal Processing, Kyoto, 2012, pp. 27692772. [Online]. Available: http://ieeexplore.ieee.org/stamp/stamp.jsp? tp=\&arnumber $=6288491$

[3] D. Ayllón, R. Gil-Pita, P. Jarabo-Amores, M. Rosa-Zurera, and C. Llerena-Aguilar, "Energy-weighted Mean Shift algorithm for speech source separation," in Proc. of IEEE Statistical Signal Processing Workshop, Nice, 2011, pp. 785-788. [Online]. Available: http://ieeexplore.ieee.org/stamp/stamp.jsp?tp=\&arnumber=5967822

[4] M. Arjona Ramírez and M. Minami, "Split-order linear prediction for segmentation and harmonic spectral modeling," IEEE Signal Processing Lett., vol. 13, no. 4, pp. 244-247, Apr. 2006. [Online]. Available: http://ieeexplore.ieee.org/stamp/stamp.jsp?tp=\&arnumber $=1605249$

[5] Y. Pantazis and Y. Stylianou, "Improving the modeling of the noise part in the harmonic plus noise model of speech," in Proc. of IEEE Int. Conf. Acoust., Speech, Signal Processing, Las Vegas, 2008, pp. 4609-4612. [Online]. Available: http://ieeexplore.ieee.org/stamp/stamp. jsp?tp=\&arnumber $=4518683$

[6] M. K. I. Molla, M. A. M. Shikh, and K. Hirose, "Time-frequency representation of audio signals using Hilbert spectrum with effective frequency scaling," in Proc. of IEEE Int. Conf. Computer and Information Technology, vol. 1, Khulna, Bangladesh, 2008, pp. 335340. [Online]. Available: http://ieeexplore.ieee.org/stamp/stamp.jsp?tp= \&arnumber $=4803077$

[7] X. Serra and J. Smith III, "Spectral modeling synthesis: A sound analysis/synthesis system based on a deterministic plus stochastic decomposition," Computer Music Journal, vol. 14, no. 4, pp. 12-24, Winter 1990. [Online]. Available: http://www.jstor.org/stable/pdfplus/ 3680788.pdf

[8] P. Polotti and G. Evangelista, "Fractal additive synthesis," IEEE Signal Processing Mag., vol. 24, no. 2, pp. 105-115, Mar. 2007. [Online]. Available: http://ieeexplore.ieee.org/stamp/stamp.jsp? tp=\&arnumber $=4117933$

[9] M. Nilsson, B. Resch, M.-Y. Kim, and W. Bastiaan Kleijn, "A canonical representation of speech," in Proc. IEEE Int. Conf. Acoust., Speech, Signal Processing, vol. 4, Honolulu, 2007, pp. 849-852. [Online]. Available: http://dx.doi.org/10.1109/ICASSP.2007.367046

[10] M. Arjona Ramírez, "Modeling the unvoiced component in the canonical representation of speech," in Proc. of DSP 2009 16th International Conference on Digital Signal Processing, vol. 1, Santorini, Greece, 2009, pp. 1-5. [Online]. Available: http://ieeexplore.ieee.org/stamp/ stamp.jsp?tp=\&arnumber $=5201232$ 
TABLE I

COMPRESSION RATIOS FOR ANALYSIS BASED UPON TFVEX VOICED MODEL, TFUNEX UNVOICED MODEL AND TFUVEX COMPOUND MODEL ALONG WITH COEFFICIENT AND PARAMETER COUNT BREAKDOWN.

\begin{tabular}{|l|r|r|r|r|r|}
\hline Model & Voiced & Unvoiced & Total & Specific ratio & Overall ratio \\
\hline \hline Signal & $421 \mathrm{k}$ & $749 \mathrm{k}$ & $1170 \mathrm{k}$ & 1.0 & 1.0 \\
\hline TFVEX & $48 \mathrm{k}$ & $749 \mathrm{k}$ & $797 \mathrm{k}$ & 8.8 & 1.5 \\
\hline TFUNEX & $421 \mathrm{k}$ & $59 \mathrm{k}$ & $480 \mathrm{k}$ & 12.7 & 2.4 \\
\hline TFUVEX & $48 \mathrm{k}$ & $59 \mathrm{k}$ & $107 \mathrm{k}$ & 10.9 & 10.9 \\
\hline
\end{tabular}

[11] R. J. McAulay and T. F. Quatieri, "Sinusoidal coding," in Speech Coding and Synthesis, W. Bastiaan Kleijn and K. K. Paliwal, Eds. Amsterdam: Elsevier Science, 1995, pp. 121-173.

[12] J. Schnitzler, J. Eggers, C. Erdmann, and P. Vary, "Wideband speech coding using forward/backward adaptive prediction with mixed time/frequency domain excitation," in Proc. of IEEE Workshop on Speech Coding, Porvoo, 1999, pp. 4-6. [Online]. Available: http://ieeexplore.ieee.org/stamp/stamp.jsp?tp=\&arnumber=781465

[13] J. D. Markel and A. H. Gray, Jr., Linear Prediction of Speech. Springer, 1976, ch. 4 and 7.

[14] L. R. Rabiner and R. W. Schafer, Theory and Applications of Digital Speech Processing. Upper Saddle River, NJ 07458: Pearson Higher Education, 2011, ch. 9.

[15] B. Bessette, R. Salami, R. Lefebvre, M. Jelínek, J. Rotola-Pukkila, J. Vainio, H. Mikkola, and K. Järvinen, "The adaptive multirate wideband speech codec (AMR-WB)," IEEE Trans. Speech Audio Processing, vol. 10, no. 8, pp. 620-636, Nov. 2002, http://ieeexplore.iee.org/stamp/ stamp.jsp?tp=\&arnumber $=1175533$.

[16] A. E. Rosenberg, "Effect of glottal pulse shape on the quality of natural vowels," J. Acoust. Soc. Am., vol. 49, no. 2, pp. 583-590, Feb. 1971.

[17] L. R. Rabiner and R. W. Schafer, Theory and Applications of Digital Speech Processing. Upper Saddle River, NJ 07458: Pearson Higher Education, 2011.

[18] H. S. Malvar, "Lapped transforms for efficient transform/subband coding," IEEE Trans. Acoust., Speech, Signal Processing, vol. 38, no. 6, pp. 969-978, June 1990. [Online]. Available: http://ieeexplore. ieee.org/stamp/stamp.jsp?tp=\&arnumber $=56057$

[19] N. S. Jayant and P. Noll, Digital coding of waveforms. Englewood Cliffs: Prentice-Hall, 1984.

[20] N. Ahmed, T. Natarajan, and K. R. Rao, "Discrete cosine transform," IEEE Trans. Comput., vol. C-23, no. 1, pp. 90-93, Jan. 1974. [Online]. Available: http://ieeexplore.ieee.org/stamp/stamp. jsp?tp=\&arnumber $=1672377$

[21] B. Boashash, Ed., Time Frequency Signal Analysis and Processing - A comprehensive reference. Elsevier, 2003, ch. 7.

[22] J.-H. Bach and J. Anemüller, "Detecting novel objects in acoustic scenes through classifier incongruence," in Proc. Interspeech, Makuhari, Chiba, Japan, 2010, pp. 2206-2209.

[23] J. S. Garofolo, L. F. Lamel, W. M. Fisher, J. G. Fiscus, D. S. Pallett, N. L. Dahlgren, and V. Zue, "TIMIT acoustic-phonetic continuous speech corpus," Linguistic Data Consortium, 1993. [Online]. Available: http: //www.ldc.upenn.edu/Catalog/CatalogEntry.jsp?catalogId=LDC93S1
[24] ITU-R, "Method for the subjective assessment of intermediate quality level of coding systems," Recommendation BS.1534-1, Geneva, Jan. 2003. [Online]. Available: http://www.itu.int/rec/R-REC-BS. $1534-1-200301-\mathrm{I} / \mathrm{e}$

[25] ITU-T, "ITU-T Software Tool Library: Software tools for speech and audio coding standardization," Recommendation G.191, Geneva, Nov. 2009. [Online]. Available: http://www.itu.int/rec/T-REC-G.191/en

[26] —-, "Perceptual evaluation of speech quality (PESQ), an objective method for end-to-end speech quality assessment of narrowband telephone networks and speech codecs," Recommendation P.862, Geneva, Feb. 2001.

[27] - "Wideband extension to Recommendation P.862 for the assessment of wideband telephone networks and speech codecs," Recommendation P.862.2, Geneva, Nov. 2005.

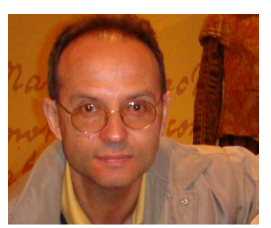

Miguel Arjona Ramírez is Associate Professor at Escola Politécnica, University of São Paulo, where he is a member of the Signal Processing Laboratory. He received the E.E. degree from Instituto Tecnológico de Aeronáutica, Brazil, and the M.S., the Dr. and the Habilitation (LivreDocência) degrees in Electrical Engineering from University of São Paulo, Brazil, in 1992, 1997 and 2006, respectively, and the Electronic Design Eng. degree from Philips International Institute, The Netherlands, in 1981. In 2008 he carried post-doctoral research in time-frequency speech analysis and coding at the Royal Institute of Technology in Stockholm, Sweden. He was Engineering Development Group Leader for Interactive Voice Response Systems (IVRs) for Itautec Informática, Brazil, where he served from 1982 to 1990. He is a Senior Member of the IEEE since 2000 and a Member of the Brazilian Telecommunications Society (SBrT). His research interests include signal compression, speech analysis, coding and recognition, speaker identification and audio analysis and coding. 